\title{
Local Anesthesia (La) without Preoperative Sedation in Tympanoplasty
}

\author{
Islam MA ${ }^{1 *}$, Mamoon $\mathrm{TB}^{2}$, Miki FU ${ }^{3}$, Chowdhury $\mathrm{NH}^{4}$, Khan $\mathrm{SR}^{5}$, Rahman $\mathrm{ASL}^{6}$ and Mohammad ${ }^{7}$ \\ ${ }^{1}$ Professor and Head, Otolaryngology-Head \& Neck Surgery, Bangladesh Medical College Hospital, Bangladesh \\ ${ }^{2}$ Assistant Registrar, ENT, Bangladesh Medical College Hospital, Bangladesh \\ ${ }^{3}$ Farid Uddin Milki, MBBS, DLO Consultant ENT, Bangladesh Medical College Hospital, Bangladesh \\ ${ }^{4}$ Assistant Professor, ENT, Bangladesh Medical College Hospital, Bangladesh \\ ${ }^{5}$ Assistant registrar, ENT, National Institute of ENT, Bangladesh \\ ${ }^{6}$ Resident Surgeon, Bangladesh Medical College Hospital, Bangladesh \\ ${ }^{7}$ Assistant Registrar ENT, Bangladesh Medical College Hospital, Bangladesh
}

*Corresponding author: Md Ashraful Islam, Professor \& Head, Department of Otolaryngology-Head \& Neck Surgery, Bangladesh Medical College Hospital, Bangladesh.

Received Date: July 16, 2020

Published Date: August 03, 2020

\begin{abstract}
Background \& Objectives: Local Anesthesia (LA) is getting popularity in comparison to general anesthesia (GA) in underdeveloped and developing countries in many otolaryngological operations. The rationale for LA is the lack of trained anesthesia staff, lack of adequate anesthesia machines and monitoring equipment. LA is safe; and ability to perform more cases in a shorter period of time. This study aimed to find out patients compliance in the operative procedures under LA without sedation.
\end{abstract}

Methods: 7905 patents underwent tympanoplsty under LA without sedation from 1999 to 2019 in the different hospital of Bangladesh. Preoperative thorough counselling with psychological motivation, advantages of LA, intraoperative surgeon patient conversation and watching surgical steps on monitor, tests of facial nerve function were explained to the patient. LA with different Adrenaline concentrations (1: 1000 to 1: 200,000 ) was used either by injection or topically. Patients' satisfaction, vertigo, drowsiness, hangover, orientation and other parameters ware recorded.

Results: Amongst 7905 patients; male $56.2 \%$ and $43.8 \%$ was female. Maximum numbers (26.3) were found between 26 year to 35 year of age. Patients' intraoperative compliance showed excellent without sedation. Operative time was short with a bloodless field. The overall safety was good and needed shortest hospital stay (Hour/day); and of course, very less expensive.

Conclusion: Patient's safety is the primary objective considered in all patients. The advantages recorded as day/hour care hospital stay, patients comfort, less bleeding, ability to assess patient's hearing during surgery and cost effectiveness.

Keywords: LA without sedation; Tympanoplsty; Safety; Cost effectiveness

\section{Introduction}

The goal of successful tympanoplsty is to create a mobile tympanic membrane or an aerated mucosal-lined middle ear space and a sound-conducting mechanism between the mobile

membrane and the inner ear fluids with a graft \pm prosthesis. The tympanoplsty is mostly done either under LA or GA. Professor D. Plester in Tubingen, Germany first started practicing by using LA 
in ear surgeries [1]. In both the anesthetic procedures have some advantages and disadvantages, but the surgeon mainly select which type of anesthesia he would love to use. LA decreases the operative time, improves hemostasis and allows intra-operative hearing assessment, minimum cost and no hospital stay. However, the method was subsequently modified by using standard adrenaline $(1: 1000)$ to injection xylocaine/lidocaine to preparing different concentration to prolong the anesthesia time and to achieve less bleeding.

A bloodless operative field is essential, because even a few drops of blood can obscure the surgical field [2]. The techniques of headup tilt 20 to 30 degrees from the operating bed facilitates avoidance of venous obstruction, normocapnia, and controlled hypotension [3]. Tympanoplsty relies some particular challenges to the surgeon. One must consider the type of procedure being performed to decide upon how best this is delivered. The patient's co-morbidities are important, and the resources in low-income settings are considered to be relevant prior to provide safe anesthesia.

Many simple procedures can be performed under LA with or without sedation. In the developing countries, this may be a safe and effective way of providing anesthesia; LA avoids the risks of GA, the requirement of equipment necessary to provide a GA safely, and even the need of an anesthetist when a suitably trained person is not available [4]. GA offers comfort to the patient and ease to the surgeon, especially for patients who cannot tolerate the procedures under LA [5]. It has been observed in the published literature that sedation is given prior LA in all tympanoplsty operation. In this study author had been doing tymanoplasty under LA without sedation for the last 20 years. In this article, a retrospective study of more than 20 years' experience of the use of LA without sedation in tympanoplasty on 7905 patients in different hospital of Bangladesh has been demonstrated. It includes description of our technique, advantages, and limitations.

\section{Objectives}

1. This study is intended to be a sharing experience of using LA without sedation.

2. It also aimed to find out patients compliance in the operative procedures without sedation.

\section{Materials and Methods}

Study design: Retrospective study

Study sample: 7905 patients with indication of chronic otitis media.

Sampling technique: Simple non-random sampling

Study period: 20 years (1999-2019)

\section{Study places:}

1) Bangladesh Medical College Hospital.

2) Popular Medical College Hospital.
3) Other district hospitals of Bangladesh.

\section{Inclusion criteria}

1) Age: 16 year to 65 year.

2) Tympanic membrane perforation

3) Chronic mucosal otitis media.

\section{Exclusion criteria}

1) Patients unfit for surgeries.

2) Chronic squamous otitis media/choleasteatoma.

3) Patients not giving consent.

\section{Procedure}

The procedure begins with preoperative patient counseling and motivation in the outpatient clinic. Many patients' question about neck stiffness for keeping still for a period of time, fear of pain and tolerability. They were reassured regarding movement of their head to other side time to time if needed and also they were taught how to cooperate during surgery. They were advised that you could go home the same day hours after operation with simple analgesics. At surgery, surgical field prepared in the standard fashion with the head on a head ring. The area was cleaned with aqueous povidoneiodine that filled the external auditory canal. The draping were applied in such a way that the patient could able to view the television monitor if he or she desired to watch the procedure.

The author used self-prepared anesthetic solution (AS) with $2 \%$ lidocaine solution with $1: 1000$ to a concentration of 1:5000 to 1:20000 which was given by injection or topically. The concentration and dose based on the age and pre-existing cardiac disease (The total amount of injected AS should not exceed $7 \mathrm{mg} /$ $\mathrm{kg}$ body weight, $20 \mathrm{ml}$ in average adult). In most patients, $12-15 \mathrm{ml}$ was needed. Injection is strictly extravascular; the syringe piston is usually withdrawn before each injection to assure extravasation. The preparation and administration of the solutions were done by the surgeon himself. Prior to infiltration, the patient was assured that he or she might experience some temporary discomfort.

LA injection technique involved four quadrant anesthetizing points in the external auditory canal. 3-5 ml AS used to inject in the temporal region for taking temporal fascia graft. Then by using 26 gauge needle, $0.5 \mathrm{ml}$ of each AS was injected in the canal in the 4 points of the external auditory canal namely 6-9-12-3 0' clock position.

The bevel of the needle is directed toward the bone, and the AS was injected subperiosteally to avoid blebs or damage to the skin. The ME mucosa was anesthetized by placing the gel foam/ small cotton ball soaked with AS through the tympanic membrane (TM) perforation. But in intact TM, $1 \mathrm{ml}$ of AS was instilled in the ME cavity once opened after elevation of the TM flap and left for 1-2min to anesthetize the tympanic plexus. In the early postoperative period, patients were asked to score their discomfort for pain, 
noise, anxiety, irrita-bility (uneasiness), and position of the body and neck by means of a questionnaire. The scores ranged from " 0 " to "4" for each question, "0" for no discomfort and "4" for extreme discomfort.

In the intraoperative \& early postoperative period, patients were asked to score their discomfort for pain, noise, anxiety, irritation (uneasiness) and position of the body and neck by a means of questionnaire. The scores ranged from " 0 " to " 4 " for each question, " 0 " for no discomfort and " 4 " for severe discomfort. Additional questions were asked about the most disturbing sensation during the operation and whether they would prefer local anesthesia for a second procedure. Per operative bleeding \& difficulties faced by the surgeon were also noted. Operating time was also recorded.

\section{Results}

In our series, LA was used in 7905 patients, 4455 (56.2\%) male and $3450(42.8 \%)$ female. Their ages ranged from 16 to 65 years (Table 1).

In the early postoperative period, patients were asked to score their discomfort for pain, noise, anxiety, irritability (uneasiness) and position of the body and neck which were noted in (Table 2).

On an average, duration of each surgeries were recorded \& presented in (Table 3).

Intra operative complications noted in (Table 4).

Postoperative complications are shown in (Table 5).

Table 1: Sample distribution of subjects according to age and gender $(n=7905)$.

\begin{tabular}{|c|c|c|c|c|c|c|}
\hline Gender & $\mathbf{1 6 - 2 5}$ years & $\mathbf{2 6 - 3 5}$ years & $\mathbf{3 6 - 4 5}$ years & $\mathbf{4 6 - 5 5}$ years & $\mathbf{5 6 - 6 5}$ years & Total \\
\hline Male & $1030(13 \%)$ & $1297(16.4 \%)$ & $935(11.8 \%)$ & $868(11 \%)$ & $325(4 \%)$ & $4455(56.2 \%)$ \\
\hline Female & $946(12 \%)$ & $1016(12.9 \%)$ & $730(9.3 \%)$ & $598(7.6 \%)$ & $160(2 \%)$ & $3450(43.8 \%)$ \\
\hline Total & $1976(25 \%)$ & $2313(29.3)$ & $1665(21.1 \%)$ & $1466(18.6)$ & $485(6 \%)$ & $7905(100 \%)$ \\
\hline
\end{tabular}

Table 2: Scores for complaints by patients $(n=7905)$.

\begin{tabular}{|c|c|c|c|c|c|}
\hline Complaints & $\mathbf{0}$ & $\mathbf{1}$ & $\mathbf{2}$ & $\mathbf{3}$ & $\mathbf{4}$ \\
\hline Anxiety & $5356(67.8 \%)$ & $1995(25.2 \%)$ & $501(6.3 \%)$ & $469(5.9 \%)$ & $14(0.18 \%)$ \\
\hline Pain & $6987(88.4 \%)$ & $870(11 \%)$ & $58(0.7 \%)$ & $16(0.2 \%)$ & $04(0.05 \%)$ \\
\hline Noise & $7694(97.3 \%)$ & $165(2.1 \%)$ & $37(0.47 \%)$ & $17(0.2 \%)$ & $02(0.03 \%)$ \\
\hline Irritation & $7697(97.4 \%)$ & $118(1.5 \%)$ & $80(01 \%)$ & $07(0.09 \%)$ & $03(0.04 \%)$ \\
\hline
\end{tabular}

Table 3: Average duration of each surgical procedure.

\begin{tabular}{|c|c|}
\hline Surgical procedure & Average required time (min) \\
\hline Tympanopasty & 35 \\
\hline
\end{tabular}

Table 4: Complications encountered during surgery $n=7905$.

\begin{tabular}{|c|c|}
\hline Complications (Perioperative) & Number of patients \\
\hline Excess bleeding & 15 \\
\hline Nausea/vomiting & 3 \\
\hline Vertigo & 0 \\
\hline Facial nerve injury/paresis & 03 (transient) \\
\hline
\end{tabular}

Table 5: Postoperative complications.

\begin{tabular}{|c|c|}
\hline Complications (Postoperative) & Number of patients \\
\hline Nausea/vomiting & 0 \\
\hline Disequilibrium/vertigo & 0 \\
\hline Facial nerve injury/paresis Sensorineural & 0 \\
\hline hearing loss Prosthesis displacement & 0 \\
\hline Wound infection & 3 \\
\hline
\end{tabular}

\section{Discussion}

Although it has been known that majority of tympanoplasty can be carried out under local anesthesia, only a small number of surgeons feel comfortable using this technique [6]. Less bleeding, cost effectiveness, postoperative analgesia, mobilization of the patient in a short time, no aspiration risk during or after the surgical pro- cedures, release of anesthetic capacity for other major procedures, and that the hearing can be tested during surgery are the advantages of local anesthesia were documented in this study which is found in other literature [7].

In our questionnaire survey, patients scored their discomfort of pain corresponding to a value ' 0 ' means no anxiety, no pain, 
no noise and irritation. ' 1 ' indicating "very mild', '2' for "mild", ' 3 ' for "moderate" and ' 4 ' for "severe". Sensation of pain is related to personal psychological status and previous experiences. Personal variations are more important than the degree of surgical trauma. Patients should be assessed meticulously by the experienced surgeon so that local anesthesia is not to be performed in unsuitable patients. The less the patient is informed, the greater his or her anxiety will be. In our series pain score was ' 0 ' in $88.4 \%$ and severe pain ' 4 ' was recorded in only $0.02 \%$. Most patients noted that they felt pain only at the beginning of injections of the local anesthetic and subsequently experienced no pain. So here it is possible by infiltrating AS properly in the external auditory canal in the fourquadrant location. The method of LA without sedation is not found in any literature.

In rating the most disturbing sensation, anxiety (0) 67.8\% of patients and $25.2 \%$ patients; the noise during the procedure complained ' 1 ' by $2.1 \%$ and irritation ' 1 ' in $1.5 \%$ of patients. It has been reported in the published literature that the intense sensation of noise and anxiety were the most common discomforts but the majority of patients preferred local anesthesia [6-8]. Adverse effects of LA did not found in many studies on local anesthesia for middle ear surgery [9-12]. In this study the results some differ form the study in the opinion that the preoperative counselling was the prime factor of getting the study result.

The choice of patients in favor of local anesthesia for a similar procedure in spite of these discomforts has various reasons. Mobilization of the patient in a short time, little pain in early postoperative period and the chance of being informed by the surgeon during the procedure are the most common reasons. In highly anxious and less-educated patients, fear of not waking up again after general anesthesia may be another reason for preferring local anesthesia.

From the surgeons' point of view, the ability to test hearing during surgery and less bleeding are the most striking advantages. In our study, hearing were assessed during the operations using free-field audiometry. In a successful hearing reconstruction, the patient heard whispered voice at $1 \mathrm{~m}$ distance. This is valuable in all ear operations aiming to improve hearing in tympanoplsty. Test of Facial Nerve (FN) action, by asking the patient to smile or close the eye lids, was a good alternative for FN monitoring in our study. It is an excellent advantage of LA without sedation.

The overall safety of LA is greater compared with GA, including elimination of the risk for laryngotracheal trauma by means of endotracheal intubation. Complications related to GA agents are avoided, especially cardiopulmonary and hepatic complications. There is less postoperative nausea and vomiting, and, in general, mobilization is early $[13,14]$. Lidocaine (xylocaine) is the main anesthetic used in our operations. It is characterized by a rapid onset and intermediate duration of action $[15,16]$. No adverse effects were found in our series. Addition of adrenaline prolongs its action and decreases its toxicity. The dose of lidocaine should not exceed $4.5 \mathrm{mg} / \mathrm{kg}$ without adrenaline, but with adrenaline it can reach $7 \mathrm{mg} / \mathrm{kg}$. Toxicity may be avoided using strict extravascular injection of AS and avoiding rubbing the skin after injection [17].

In our study, it was found that the time of action of lidocaine was nearly doubled if adrenaline 1:1000 was added and increased by 2-fold again with 1:20 000 adrenaline. This is in agreement with the opinion of Neal et al. $[18,19]$, who stated that adrenaline causes vasoconstriction to arteries, reducing bleeding, and delays the absorption as well resulting doubling the duration of anesthesia. We also found that prolongation of lidocaine action which was also related to the site of injection. The cost-effectiveness of LA is agreed upon by many authors $[19,20]$. The shorter operative time took average 35 minutes, shorter hospital stay 30 minutes to hour and saving cost of GA/sedation all contribute to the low cost of LA cost effectively without sedation in this study. In a previous study carried in Al Azhar University Hospitals, the anesthetic drugs in LA cost less than $20 \%$ of the cost of anesthetic drugs of GA [21]. Because of the low cost, early recovery, and shorter hospital stay (day cases), LA without sedation was used successfully by the author in tympanoplasty in different tertiary level hospitals.

\section{Conclusion}

Tympanoplsty under local anesthesia without sedation is safe, feasible and cost-effective. It causes less bleeding and is well tolerated both by patients and by the surgeon. Good patient selection and preoperative counselling are the most important factors for the success of LA without sedation. The majority of patients leave hospital within hours on the same day.

\section{Acknowledgement}

None.

\section{Conflict of Interest}

No conflict of interest.

\section{References}

1. Plester D (1963) Myringoplasty methods. Arch Otolaryngol 78: 310316.

2. El-Begermy MA, El-Begermy MM, Rabie AN, EM Ezzat A, Sheesh AAK (2016) Use of local anesthesia in ear surgery: technique, modifications, advantages, and limitations over 30 years' experience. The Egyptian Journal of Otolaryngology 32(3): 161-169.

3. Caner G, Olgun L, Gültekin G, Aydar L (2005) Local anesthesia for middle ear surgery. Otolaryngol Head Neck Surg 133(2): 295-297.

4. Hollinshead WH (1982) Anatomy for sur geons: Volume 1: The head and neck. Philadelphia: Lippincott Williams \& Wilkins.

5. Peuker ET, Filler TJ (2002) The nerve supply of the human auricle. Clin Anat 15: 35-37.

6. Edussuriya B, Goonasekera CD, Rajapakse M, Rajapakse VP, Jayasooriya D (1997) Middle Ear Surgery Under Local Anaesthesia and Sedation. Ceylon Med J 42(2): 75-77.

7. Yung MW (1996) Local anesthesia in middle ear surgery: survey of patients and surgeons. Clin Otolaryngol 21: 404-408.

8. Lancer JM, Fisch U (1998) Local anesthesia for middle ear surgery. Clin Otolaryngol 1988 13: 367-374. 
9. Andreassen UK, Larsen CB (1990) [Anesthesia in ear surgery. A source economicl analysis and patient assessment of general anesthesia versus local anesthesia in ear surgery]. Ugeskr Laeger 28: 152: 1595-1597.

10. McLeod IK, Gallagher DJ III, Revis DR, Seagle MB (2008) “Local Anesthetics" eMedicine.

11. Cousins MJ, Bridenbaugh PO (1998) Neural blockade in clinical anesthesia and management of pain, ( $3^{\text {rd }}$ Edn.), Philadelphia: Lippincott-Raven, USA

12. Abdul-Latif RM (2012) Stapedectomy data base study [MS degree in OLR]. Faculty of Medicine, Ain Shams University, Cairo, Egypt.

13. Martha R, Amoroch C (2000) Anesthesia for Tympanomastoidectomy Anesthesiology pp: 281-285.

14. Sarmento KM, Tomita S (2009) Retroauricular tympanoplasty and tympanomastoidectomy under local anesthesia and sedation. Acta Otolaryngol 129(7): 726-728.

15. Pöntinen PJ (1978) Cardiovascular effects of local adrenaline infiltration during halothane anaesthesia and adrenergic beta-receptor blockade in man. Acta Anaesthesiol Scand 22(2): 130-144.
16. Becker DE, Reed KL (2006) Essentials of local anesthetic pharmacology. Anesth Prog 53(3): 98-108.

17. Jackson D, Chen AH, Bennett CR (1994) Identifying true lidocaine allergy. J Am Dent Assoc 125(10): 1362-1366.

18. Abdellatif AA, Elkabarity RH, Hamdy TA (2012) Dexmedetomedine vs midazolam sedation in middle ear surgery under local anesthesia: effect on surgical field and patient satisfaction. Egyptian Journal of Anaesthesia 28: 117-123.

19. Neal JM, Bernards CM, Butterworth JF (4 ${ }^{\text {th }}$ Edn.) (2010) di Gregorio G, Drasner K, Hejtmanek MR (Eds.), ASRA practice advisory on local anesthetic systemic toxicity. Reg Anesth Pain Med 35(2):152-161.

20. Nader ND, Simpson G, Reedy RL (2004) Middle ear pressure changes after nitrous oxide anesthesia and its effect on postoperative nausea and vomiting. Laryngoscope 114(5): 883-8866.

21. Ghanem AM (2003) Cartilage tympanoplsty (Type1) by local anesthesia 'Costs and Results' Thesis Submitted for partial Fulfillment of MS degree in ORL, Al Azhar University, Cairo, Egypt. 